\title{
Pediatric liver diseases: current challenges and future perspectives
}

\section{Claudia Della Corte, Antonella Mosca, Andrea Vania, Arianna Alterio, Anna} Alisi \& Valerio Nobili

To cite this article: Claudia Della Corte, Antonella Mosca, Andrea Vania, Arianna Alterio, Anna Alisi \& Valerio Nobili (2015): Pediatric liver diseases: current challenges and future perspectives, Expert Review of Gastroenterology \& Hepatology, DOI: 10.1586/17474124.2016.1129274

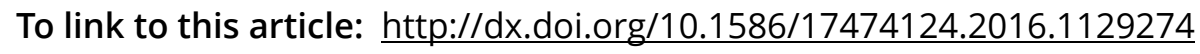

Accepted author version posted online: 05

Dec 2015.

Submit your article to this journal $\pi$

View related articles ¿

View Crossmark data $₫$ 
Publisher: Taylor \& Francis

Journal: Expert Review of Gastroenterology \& Hepatology

DOI: $10.1586 / 17474124.2016 .1129274$

Pediatric liver diseases: current challenges and future perspectives

Claudia Della Corte ${ }^{1}$, Antonella Mosca ${ }^{2}$, Andrea Vania ${ }^{2}$, Arianna Alterio ${ }^{1}$, Anna Alisi ${ }^{3}$ and Valerio Nobili $^{1}$

1 Hepato-Metabolic Department, Bambino Gesù Children's Hospital, IRCCS, Rome, Italy.

2: Center of Dietetics and Nutrition, Pediatric Clinic, 'La Sapienza' University, Rome, Italy.

${ }^{3}$ Liver Research Unit, Bambino Gesù Children's Hospital, IRCCS, Rome, Italy.

\section{Corresponding Author}

Claudia Della Corte, MD

Hepatometabolic Department, Liver research Unit

Bambino Gesù Children's Hospital, IRCCS,

P.le S. Onofrio, 4-00165- Rome, Italy.

Electronic address: claudia.dellacorte@opbg.net

+39 06/68592077 


\section{Summary:}

Chronic liver diseases in children represent a rising problem with significant effects on public health. In fact, several pediatric liver diseases are precursors of adult chronic hepatopathies, cirrhosis and hepatocellular carcinoma. The prevalence of liver diseases in children is unknown. In the USA, every year, 15,000 children are hospitalized for liver diseases, but these disorders continue to be under-recognized or diagnosed late. The main reason is due to the frequent absence of symptoms in the vast majority of liver diseases, especially in the early stages. In the last few decades several advances have been made in understanding the pathogenesis of liver diseases, permitting the discovery of new therapeutic targets to treat liver diseases, thus improving the natural history of these disorders. In this article we discuss the most recent advances in the understanding of the pathogenesis, diagnosis and treatment of the most frequent pediatric liver diseases.

\section{Introduction}

Chronic hepatobiliary diseases represent a relatively rare disorder in children. However, the burden of chronic hepatopathies, defined as impact of liver disease on the global health, including its frequency, its effects on longevity, morbidity, quality of life and finance, with direct health care expenditures and indirect costs, is very important.

The exact prevalence of pediatrie liver diseases is unknown. Liver disease is not cited in the first 15 causes of death in children; the only data available reported that each year approximately 15.000 pediatric patients are hospitalized for liver diseases in USA [1]. European epidemiological pediatric data are not available so far; for adulthood, it is reported that 29 million people are affected by liver diseases and that cirrhosis and end-stage liver diseases are responsible for around 170.000 deaths every year, representing the $12^{\text {th }}$ most common cause of death [2]. Globally, the number of annual liver cancer and cirrhosis death rose in 2010, with 33 per cent more than in 1990 [3], according to the first-ever World Health Organization (WHO) study of liver disease mortality. 
Pediatric hepatopathies are particularly important because of several pediatric diseases are precursor of adult chronic liver diseases, cirrhosis and hepatocellular carcinoma; therefore, treatment and prevention in pediatric setting would be highly cost-effective. Based on these data, governments are now considering liver diseases as a public health priority with urgent need to develop non-invasive tests to screen for early stage of fibrosis in order to promptly treat and stopping or slowing the progression of liver damage.

Instead, liver diseases in children are often under-recognized or late diagnosed. This under-diagnosis is mainly due to the frequent absence of symptoms in the vast majority of liver diseases, especially in the early stages. Moreover, different liver diseases often show a similar pattern of presentation. In fact, generally, hepatic cells react to different injuries with similar responses, with frequently monomorphic clinical and laboratory signs. The types of liver injury are generally divided into two patterns: cholestatic or hepatocellular damage, with sometimes overlap pattern. Moreover, the causes of liver diseases in children are manifold, and many of these are rare disorders. It is important to remember in the diagnostic work-up of suspected hepatopathy in children that many liver diseases are associated with specific age groups, such as for example - biliary atresia in the first months of life or Wilson disease after 3-5 years of age (see Table 1). Again, in the difficult diagnosis of liver disease it is important to consider the frequent absence of symptoms, with only sometimes non-specific signs, such as fatigue, loss of appetite, vague abdominal pain, hepatomegaly or pruritus (Table 2). Although many advances have been made in the diagnostic tools for pediatric liver disease (laboratory assessment, specific imaging techniques), percutaneous liver biopsy remains the standard procedure to obtain liver tissue for histopathological evaluation and a valuable tool in the diagnosis, prognosis and management of many liver diseases. Recently, the Hepatology Committee of European Society of Gastroenterology, Hepatology And Nutrition (ESPGHAN) has published a position paper on liver biopsy, focusing on indication, contraindications and type of procedure in principal pediatric hepatopathies [4].

Many advances have been made in the knowledge of pathogenesis of many liver diseases and based on these discoveries novel emerging treatments have been proposed, changing the natural history and epidemiology of disorders. Moreover, the advances in transplant procedure have opened new possibilities for providing care for several hepathopathies, previously only partially treatable. 
In this manuscript, we discuss the most recent advances in knowledge of pathogenesis, histological features, diagnosis and treatment of principal pediatric chronic liver diseases.

\section{NAFLD}

Non-alcoholic fatty liver disease (NAFLD) represents the leading cause of chronic liver disease in children, as a direct consequence of the worldwide epidemic obesity in children. The exact prevalence of NAFLD/NASH is unknown, but the available data reported that approximately 3-10\% of general pediatric population is affected by NAFLD and this percentage increases up to 70\% in morbidly obese children [5]. The histopathological scenario of NAFLD ranges from simple triglycerides accumulation in the hepatocytes to more advanced forms, characterized by various degrees of inflammation and fibrosis (non-alcoholic steatohepatitis - NASH).

In the last decade, many advances have been made in the knowledge of pathogenetic mechanisms involved in NAFLD/NASH and several intriguing novel therapeutic approaches have been proposed. The known theory of "two hits hypothesis", in which the accumulation of liver fat (first hit) increased the vulnerability of liver cells to "second hits", such as insulin resistance and oxidative stress, causing the disease progression, has been now exceeded. Current evidence suggests the central role of "lipotoxicity", respect to simple triglycerides accumulation, in inducing the hepatocytes dysfunction leading to the progression of liver injury to NASH [6]. Some studies have, in fact, demonstrated that triglycerides accumulation in the hepatocytes, due to increased synthesis or reduced export of lipids, is unable, by itself, to determine the progression of liver damage. Probably, triglycerides accumulation occurs in parallel with the generation of these lipotoxic metabolites, which act as reactive oxygen species (ROS), triggering necroinflammation changes typical of NASH [6]. Insulin-resistance and inflammatory cytokines disarray are closely involved in this pathogenetic mechanism, inducing a variety of intra- and inter-cellular signaling cascades leading to fibrosis and advanced liver damage [6]. Moreover, an interesting interaction between liver and gut has been proposed as important in the pathogenesis of chronic hepatitis, such as NAFLD. Recent studies have demonstrated that NAFLD is associated with increased intestinal permeability (IP) and small intestinal bacterial overgrowth (SIBO), and that these factors are associated with the severity of hepatic disease [7]. Based on these novel findings, actually NAFLD is considered a "multi-hits" parallel disorder, in which genetic, epigenetic and 
environmental factors strictly interact causing the onset and progression of liver injury [6,9]. In addition, recent studies have highlighted the importance of genetic factors in the development of NAFLD/NASH, identifying single nucleotide polymorphisms (SNPs) in specific genes involved in lipid metabolism (Lipin 1 - LPIN1, patatin-like phospholipase domain containing-3 - PNPLA3), oxidative stress (superoxide dismutase 2 - SOD2), insulin signalling (insulin receptor substrate-1 - IRS-1) and fibrogenesis (Kruppel-like factor 6KLF6), which confer a genetic predispositions to more severe forms of liver damage $[9,10]$. Recently, a non-invasive kit to screen for four-polymorphisms related to NASH has been proposed to recognize children at high risk of disease progression, but further and larger studies are needed before implementing the use of such genetic tests in clinical practice on large scale [11].

NAFLD is generally asymptomatic and the diagnosis is made after the evidence of hyperechogenic liver at ultrasound or in case of abnormal levels of liver enzymes. To make the diagnosis of NAFLD other causes of hepatic steatosis (viral hepatitis, autoimmune hepatitis, Wilson's disease, $\alpha 1$-anti-trypsin, celiac disease) in children should be ruled out by appropriate tests. According to recent recommendation of Hepatology Committee of ESPGHAN, NAFLD should be suspected in all overweight or obese children and adolescents with increased waist circumference aged over 3 years, especially if there is a NAFLD history in relatives [12]. The first diagnostic step in these children should be abdominal ultrasound and liver function tests, followed by the exclusion of other liver diseases [12]. To date, liver biopsy remains the "imperfect gold standard" for diagnosis, and it is the only diagnostic tool to distinguish between NASH and simple steatosis, and to determine the severity of liver damage [12]. Several non-invasive biomarkers, both laboratory that imaging tools, of NAFLD/NASH have been proposed in the last years [13] . Oxidative stress markers, inflammatory cytokines and adipokines have been tested as potential non-invasive indices of fibrosis both in adults than children, but none of these tests have proved totally satisfactory [13]. Actually, these markers need further validation and are confined to research purposes because of their costs and limited availability. Identifying and validating potential novel non-invasive biomarkers of NAFLD and NASH is a central area of research in the near future. Regarding imaging techniques, hepatic ultrasonography remains the most commonly used modality because of safe, non-invasive, less expensive and user-friendly. However, ultrasound is unable to detect fat infiltration below $30 \%$ or to assess disease severity or the presence or absence of NASH. Hepatic magnetic resonance (MRI) has a number of advantages, including operator 
independence and reproducibility. Unfortunately, similarly to ultrasound, MRI cannot distinguish NASH from simple steatosis nor detect the presence of fibrosis and, moreover, its high costs and need of sedation, especially in younger children, limit significantly its use. Transient elastography (TE) is a novel ultrasonographic technique, which employs ultrasound-based technology to measure liver stiffness (LSM), and has been validated for use mainly in adult patients with chronic hepatitis C and chronic hepatitis B. However, recent studies had shown good results in adults with NAFLD [13]. The main limitations of TE are represented by its inaccuracy in obese subjects and by the limited data currently available for pediatric NAFLD patients. Further validation of TE is necessary before it can be routinely adopted for the evaluation of NAFLD in childhood $[14,15]$. Novel therapeutic strategies have been tested in the last years with interesting results [16]. The drugs initially used in treatment of NAFLD, such as anti-oxidants (vitamin E), insulin-sensitizing (metformin) and cytoprotective agents (ursodeoxycholic acid - UDCA) have given disappointing results, with only limited effects, and, therefore, to date there is no definitive treatment for pediatric NAFLD. Regarding vitamin E, the largest adult RCT, the PIVENS trial, showed significant improvements in the aminotransferase levels, hepatic steatosis and lobular inflammation in the vitamin $\mathrm{E}$ group compared to the placebo group [17]. In 2012, the American Association for the Study of Liver Diseases (AASLD) suggested the use of vitamin E (800 UI/daily) in adult non-diabetic patients with biopsyproven NASH [18]. In pediatric setting, in the TONIC trial, the largest pediatric RCT, significant differences between the vitamin $\mathrm{E}$ and placebo groups in improving alanine aminotransferase (ALT) levels were not found; however, an improvement in hepatocytes ballooning, without differences in steatosis or lobular inflammation, was observed between the two groups ([19]. To date, the available data for vitamin $\mathrm{E}$ are not convincing enough to recommend the use of this drug in pediatric NAFLD [20]. Larger well-designed RCTs with adequate power and duration concentrating on the histological endpoints in children are still needed to better define the efficacy of vitamin E in pediatric NAFLD[20]. Interesting data have been instead reported in children for omega-3 fatty acid, mainly for docosaesaenoic acid (DHA), with beneficial effect on metabolic parameters (insulin-sensitivity and triglycerides level) and on liver steatosis, at echographic and histological examination[21-24]. Recently, Janczyk and coworkers reported the results of their multicenter, double-blind, placebo-controlled, RCT in which 76 pediatric patients with NAFLD received weight-based omega-3 fatty acids (DHA and EPA in 3:2 proportion, 450-1300 mg daily) or placebo twice daily for 24 
weeks. In this study, supplementation with omega-3 fatty acids was not superior to placebo in inducing significant reduction in ALT levels or in improving hepatic steatosis on ultrasonography [25]. Therefore, future larger studies for longer duration with histological end-points are required to prove the efficacy of omega-3 on NAFLD in children.

Bariatric surgery represents a new frontier of the treatment of morbid obesity with co-morbidities. In the recent years, the number of adolescents undergoing to bariatric surgery in America has increased fivefold with positive effect on metabolic parameters [26]. Recently, bariatric surgery is becoming a useful option in patients with NASH, also in adolescents. In 2015, the Hepatology Committee of ESPGHAN has proposed a position paper about the inclusion criteria for bariatric intervention in adolescents with morbid obesity [27]. Many studies have shown that bariatric surgery improved metabolic parameters and histologic features of $\mathrm{NASH}$; in a recent metanalysis, Mummadi et al reported a high rate of improvement of histological features of NASH (90\% for steatosis and 70\% for fibrosis) after bariatric surgery [28]. However, despite these encouraging results, it should be highlighted that the paucity of long-term outcome information regarding nutritional deficiency, growth and developmental impact of bariatric surgery in children and adolescents makes the role of bariatric surgery in the pediatric patients still controversial [27,29]. Another useful alternative is represented by temporary devices for inducing weight loss, such as intragastric balloon, in which a significant weight loss could induce a positive effect on metabolic parameters related to metabolic syndrome and liver alteration [30] 


\section{Chronic viral hepatitis: the changes of epidemiology and the new drugs}

The epidemiology of viral hepatitis in children has radically changed in the last years, due to improved hygiene measures, health status and blood supply, and to introduction of universal vaccination for hepatitis B. Moreover, recently, new antiviral drugs have been introduced in the pharmacological armamentarium, mainly for hepatitis $\mathrm{C}$, with encouraging results in adults and in pediatric pilot studies. The association between improved prevention strategies and new effective drugs could lead in the coming years to a significant reduction in the cases of chronic viral hepatitis and their medium and long-term complications (cirrhosis and hepatocellular carcinoma-HCC).

\section{Hepatitis B}

Despite the conspicuous reduction in prevalence of chronic hepatitis B infection observed in the last two decades, HBV hepatitis continues to represent a major global health problem [31]. It has been estimated that actually 2 billion people worldwide are affected by HBV infection and that about 600.000 persons died every year for hepatic consequences of chronic hepatitis B, such as cirrhosis and HCC [32].

Since 1990, a comprehensive strategy was recommended in order to eliminate of HBV transmission in Industrialized Countries based on:

- Universal vaccination of infants in the first months of life (according to local vaccination programs)

- Routine screening for HBV of all pregnant women and immunoprophylaxis at birth to infants born by infected women

- Routine vaccination of children/adolescents

- Vaccination of adults at increased risk for infection (health-care workers, patients affected by chronic diseases, etc)

Though this program in the last twenty years the prevalence of HBV infection is dramatically decreased and some previously high-preyalence countries (Hepatitis B surface antigen (HBsAg) positivity higher than 8\%), such as China and Taiwan, have become at intermediate or low prevalence (HBsAg 2-7\% or <2\%, respectively) $[33-37]$.

Chronic hepatitis B (CHB) had generally a benign course in children, even if in one old cohort study has been reported that $3-5 \%$ and $0.01-0.03 \%$ of chronic carriers develop cirrhosis or hepatocellular carcinoma, respectively, before adulthood $[38,39,40]$. Considering the average life expectancy of pediatric patients, the risk for HCC rises to 9-24\%, with an incidence of cirrhosis of 2-3\% per year during lifetime [41][42].

The natural history of chronic hepatitis B in children is generally favorable; the vast majority of children are asymptomatic with normal growth and psychological development. Three phases of chronic hepatitis B have been described: the immune-tolerant state, typical of pediatric age, characterized by high viral replication, positive $\mathrm{HBeAg}$, and normal levels of aminotransferases; the immune active state, described by active 
inflammation and elevated aminotransferases, which can progress into cirrhosis; the inactive carrier state, marked by seroconversion to anti-HBe and low viral replication with favorable long-term outcome [43].

Differently from adults, clear guidelines for treatment of pediatric CHB are lacking and a panel of experts developed the available indication [44]. Treatment should be considered in children in immune active state; the presence of inflammation and fibrosis at liver biopsy (Ishak), not mandatory but suggested before treatment, and positive family history for HCC, are considered additional criteria for pharmacological treatment. The ideal objective of CHB treatment is to induce HBsAg clearance, reducing the risk of disease progression with improvement of long-term survival and quality of life [38]. When HBsAg seroclearance is not achieved, sustained off-therapy suppression of viral replication with subsequent reduction of inflammation and ALT levels, and/or durable anti-HBe seroconversion, are considered a good end-points, being associated with improved prognosis, including decreased risk of HCC [38]. The FDA has approved the use of five medications for treating hepatitis B in children: Interferon-alpha (IFN- $\alpha$ ) and four nucleoside analogues (Lamivudine, Tenofovir, Entacavir, and Adefovir dipivoxil). IFN- $\alpha$ can be used in children older than 12 months of age, lamivudine starting at 3 years of age, adefoyir and tenofovir in children aged 12 years and older and entecavir starting from 16 years of age. IFN- $\alpha$ has been the first drug used for treatment of CHB in children; the long-term studies have demonstrated that IFN- $\alpha$ accelerate HBeAg seroconversion, but treated and untreated patients had a similar rate of HBeAg clearance at long-term follow-up [44]. Licensing of highly effective nucleoside analogues (NA) for older children and adolescents has opened new possibilities of treatment. However, the risk of emergence of drug resistant strains represents a major longterm issue for young patients. In this contex, promising data have been emerged in children for tenofovir, a NA with an excellent safety profile and absent drug resistance. Murray et al have demonstrated in adolescents (12-17 years) a virologic and biochemical response in $89 \%$ and $74 \%$ of treated patients, respectively [45]. Considering the good safety profiles and absence of emergence of drug resistance strain at 72 weeks of therapy, tenofovir seems to be an interesting agent for children, even if long-term follow up studies are needed to confirm these data. Moreover, recent evidence reported the efficacy and security of tenofovir not only in children, but also in pregnancy, in an attempt to reduce a mother-to-infant HBV transmission. Two recent prospective multicenter trials demonstrated that the treatment with TDF for highly viremic mothers significantly decreased perinatal HBV transmission without side effects on obstetric and neonatal parameters $[46,47]$.

\section{Hepatitis $\mathbf{C}$}

Following the accurate screening of blood products and organ donors, the prevalence of HCV has been significantly reduced. Today, it is estimated that 170 million people worldwide are affected by chronic hepatitis C [48]. In children the main source of infection is the vertical transmission, mother to child, which occurs in about $5 \%$ of cases. The spontaneous clearance of the virus is observed in $20 \%$ of children infected 
in the first 3 years of life, with rate of chronicity of infection in $80 \%$ of cases. Similarly to CHB, the principal risk related to $\mathrm{HCV}$ infection is the development of cirrhosis and its complication during life.

The natural history of chronic hepatitis $\mathrm{C}(\mathrm{CHC})$ in children is usually benign, even if pediatric $\mathrm{CHC}$ is considered a slowly progressive disease with accumulating liver damage following the years [49]. By the end of adolescence, about $2 \%$ of patients develop cirrhosis [50].

Assessment of liver disease severity is generally recommended prior to therapy in adults and children [51]. In adults, alternative non-invasive methods, such as liver stiffness measurement (LSM) and well-established panels of blood biomarkers, are routinely used instead of liver biopsy to assess liver disease severity prior to start therapy. Conversely, in children, these non-invasive tests are not commonly used in clinical practice; the actual European and American pediatric guidelines for the management of $\mathrm{CHC}$ in children suggest to forego pre-treatment liver biopsy in children with $\mathrm{HCV}$ genotypes 2 or 3 who have a high (>80\%) probability of achieving a virological response with presently available treatments [49]. In the other genotypes ( 1 or 4 ), in which about $50 \%$ of virological response has been described, pre-treatment liver biopsy remains useful for decision making; if fibrosis is mild, expectant management (awaiting better regimens) is justifiable.

The combined therapy (Pegylated (PEG)-interferon (IFN) plus ribavirin represents the standard treatment for children aged 3 years and older [52]. This regimen is effective in obtaining sustained viral rates in 55\% of children chronically infected with genotype 1-4 and in 90\% of genotypes 2-3. Recently, novel different direct-acting antiviral agents (DAAs) are emerged for treatment of chronic HCV infection in adults [53]. These agents block viral replication by directly inhibiting one of several steps of the HCV lifecycle. The DAAs include NS3-4A protease inhibitors, nucleoside analogue inhibitors of the HCV RNA-dependent RNA polymerase (RdRp) and NS5A, another protein inhibitors.

The first used NS3-4A protease inhibitors were telaprevir and boceprevir. Their use in monotherapy resulted in initial decline in plasma HCV-RNA concentration with unfortunately rapid selection of resistant variants and viral breakthrough [54]. Better results in terms of sustained virological response (70-80\% in genotype 1) and risk of resistance were reported for the association of telaprevir or boceprevir with PEG-IFN plus ribavirin [55]. An important limitation of this triple therapy is the important adverse effects, mainly hematological. Similarly to NS3-4A proteinase inhibitors, also NS5A inhibitors, as daclatasvir, showed a rapid reduction in viral load with frequent onset of resistance [56].

NS5B inhibitors interfere with viral replication by binding to the NS5B RNA-dependent RNA polymerase. Sofosbuvir is the most promising drug of this class of DAAs [57]. The use of Sofosbuvir associated to Ribavirin in adults showed interesting results with optimal sustained virological response (70-90\%) in genotype 2-3 and good safety profile. For previously untreated genotype 1 patients, sofosbuvir plus ribavirin resulted in $84 \%$ virological response [56][58]. These results, associated with its IFN-free regimen, the good 
safety profiles and the once-daily administration, make sofosbuvir a suitable drug also in pediatric setting [59], but specific pediatric studies are needed.

\section{Wilson's disease}

Wilson's disease (WD) is a genetic copper storage disorder, transmitted as an autosomal recessive trait, caused by mutation in ATP7B gene (13q), causing a progressive toxic accumulation of copper in the body [60].

WD is present worldwide with a frequency ranging from 1: 30.000 to $1: 50.000$, depending on the different ethnic groups evaluated. It is estimated that 1:90 people is a carrier of the disease. Regional clustering of WD has been identified as in an Italian region, Sardinia, in which the prevalence is higher, about 1:2700 [61].

Wilson's disease is an important liver disorder in which a prompt diagnosis and initiation of therapy could significantly change the natural history of the disorder and the quality of life for the patients. In fact, if early and adequately treated, WD has a good quality of life and a favorable outcome. If WD is not recognized, the progression of hepatic and neurologic damage can be very rapid and end stage liver disease can occur with need of liver transplantation [62].

In 2003, Ferenci et al. proposed a diagnostic score for WD in children including clinical, biochemical, histological and molecular parameters [63]. The first step in the diagnostic work-up for WD is represented by serum ceruloplasmin levels, which are characteristically reduced in WD. However, it is known that in $20 \%$ of pediatric and adult patients with WD ceruloplasmin levels may be normal. Furthermore, on the other hand, low levels of ceruloplasmin could be observed also in other hepatic disorder, such as decompensated liver disease or in other chronic hepatopathies, as congenital disorder of glycosylation [60][64].

Recently, relative exchangeable copper (REC) has been proposed and evaluated as a new faster diagnostic test for WD. REC corresponds to the labile fraction of copper bond mainly to albumin. An increased REC reflects a blood and tissue copper overload that occurs after saturation of hepatocytes. Further investigations are needed to verify the promising data about this diagnostic approach [65].

Another useful diagnostic test is the basal urinary copper (CUB), which is related to the age at diagnosis and to the copper overload. Values $>100 \mu \mathrm{g} / 24$ hours are diagnostic of WD, but several data have demonstrated that lower values should be considered diagnostic in pediatric setting, suggesting a new pediatric cut-off at > 
$40 \mu \mathrm{g} / 24 \mathrm{~h}$. In addition other diagnostic test is measurements of urine excretion of copper after a challenge penicillin sheets $(\mathrm{CuPCT})$, the values $>1600 \mu \mathrm{g} / 24 \mathrm{~h}$ are considered diagnostic [66]. Hepatic copper accumulation is the histological mark of WD; liver copper content exceeding $250 \mu \mathrm{g} / \mathrm{g}$ dry weight is diagnostic for WD. The other qualitative histological evaluation of hepatic copper is suggestive but not diagnostic of WD [67]. Genetic tests searching for mutations in ATP7B gene conclude the diagnostic process of WD. The presence of mutations on both chromosomes permits a definitive diagnosis of WD, while the absence of genetic mutations cannot exclude the diagnosis in the presence of clinical and laboratory signs [68]. To date, it has reported over 520 mutations in ATP7B gene in the Human Genome Mutation Database [69].

Medical therapy is mandatory for the whole-life and arbitrary discontinuation is particularly worrisome, exposing to the risk of acute liver failure and death. Conventional medical treatment for children with WD is represented by copper chelators (penicillamine and trientine) and zinc. The chelating agents mobilize intracellular copper in circulation promoting the urinary excretion of copper, while zinc causes a reduced intestinal absorption of copper and simultaneously reduced the damaging effects of free copper in the hepatocytes. Recently it was reported that about 30\% of WD patients adequately treated since childhood with the available drugs do not exhibit complete normalization of liver enzymes but have a good quality of life and a favorable outcome [70].

The treatment of pediatric patients is quite different from adulthood. In children with mild liver disease and in asymptomatic children, monotherapy with zinc seems to be effective in to treatment of WD at the onset and during the course of the disease, as maintenance therapy. The children unresponsive to zinc or with more severe hepatic injury should be treated with chelators.

In conclusion, the pharmacological available options for pediatric WD seem to be effective in the vast majority of cases. To date, the research is looking for novel therapeutic strategies for WD in addition to conventional therapy. The main field of interest is the identification of new molecular strategies able to restore the function of mutated protein, decreasing the toxic copper accumulation. 


\section{Autoimmune hepatitis}

Autoimmune hepatitis is a progressive inflammatory disorder of the liver, characterized by hypertransaminasemia, high level of immunoglobulin $\mathrm{G}(\mathrm{IgG})$ and by the presence of autoantibodies. The main histological feature is the interface hepatitis in the absence of a known etiology [71]. Based on autoantibodies pattern, two type of AIH have been described in children: type 1, with seropositivity for smooth muscle antibodies (SMA) and/or antinuclear antibodies (ANA), and type 2, with positivity for liver kidney microsomal type 1 antibodies (anti-LKM1) or for liver cytosol type 1 antibodies (anti-LC1) [72]. These two types differ for age at onset, type of presentation, response to treatment withdrawal.

The prevalence of AIH varies between different areas, but generally it is about 2 cases per 200000 pediatric patients. Recent data showed an increase of the prevalence of this disease from $3 \%$ to $12 \%$ in the last decade in pediatric population [73]. AIH remains today an under-diagnosed disorder, and some decompensated liver cirrhosis due to 'cryptogenic hepatitis' are in reality secondary to autoimmune liver diseases [74]. The diagnosis of AIH is in fact based on the simultaneous combination of clinical, serological and histological features and not on a definite diagnostic test. Moreover, the clinical presentation of AIH are highly variable in children, ranging from an acute hepatitis (40\% of cases) with the risk of progression to liver failure (especially in AIH type II), and an insidious onset (25-40\% of cases), with progressive fatigue, intermittent jaundice, anorexia, and weight loss, lasting several months before diagnosis. In a minor group of patients, about $10 \%$, AIH may begin with complications of advanced liver disease, such as portal hypertension, cirrhosis, hypersplenism [74][75]. Therefore, a high index of suspicion should be held, in order to prompt diagnose and treat $\mathrm{AIH}$, reducing the risk to develop serious complications and end stage liver diseases. It has been demonstrated that the prognosis is related to the presence of cirrhosis at the initial liver biopsy; the presence of cirrhosis on initial liver biopsy has no impact on the overall survival rate, as well as on the survival free of liver transplantation [76].

The goal of immunosuppressive treatment is represented by the prevention of disease progression, maintaining on long term the lowest dosage of drugs, avoiding or reducing the need of liver transplantation. The standard therapy in children is represented by corticosteroids, which are effective in near $80 \%$ of cases, associated in the vast majority of cases to Azathioprine [77]. Cessation of treatment is considered if a liver 
biopsy shows minimal or no inflammatory changes after 1 year of normal liver function tests. However, it is advisable not to attempt to withdraw treatment within 2 years from diagnosis or during or immediately before puberty, when relapses are more common. Despite the efficacy of standard immunosuppressive treatment, severe hepatic decompensation may develop, even after many years of apparently good biochemical control, leading to transplantation $10-15$ years after diagnosis in $10 \%$ of the patients. Inpatients (up to10\%) in whom standard immunosuppression is unable to induce stable remission or who are intolerant to azathioprine, mycophenolatemofetil at a dose of $20 \mathrm{mg} / \mathrm{kg}$ twice daily has been successfully used [78][79]. In case of persistent no response or of intolerance to mycophenolate mofetil, the use of calcineurin inhibitors (cyclosporin A or tacrolimus) should be considered [80][81]. New drugs, such as budenoside, are being evaluated for treatment of AIH in children with a reduction in steroid side-effects [82][83]. The available data seem to demonstrate that budesonide in association with azathioprine therapy was capable of maintaining remission with fewer side effects respect to prednisone [84,85]. However, budesonide may be less effective in inducing remission. To date, budesonide could be considered a valid alternative in selected non-cirrhotic patients who are at risk of adverse effects from steroids, but further larger studies are needed to define its efficacy in children with AIH [86].

A particular subtype of AIH is the seronegative form, in which none of the typical autoantibodies are detectable. In these cases the diagnosis can be more difficult and a high index of suspicion should be held. The correct diagnosis of this form is based on the combination of serological pattern (generally elevation of IgG), typical histological findings and appropriate investigations to carefully exclude other possible causes of liver disease. If promptly diagnosed and treated, these AIH subtypes respond positively to standard immunosuppressive therapy [87].

\section{Expert commentary}

Even if generally considered a rare disorder, chronic liver diseases in children represent a growing problem with important effects on public health. In fact, advances in the management of children with chronic liver disease have enabled many to survive into adulthood with or without their native livers, so that the most common of these conditions are becoming increasingly common in adult hepatology practice. Therefore, 
creating a standardized transition program represents an important advance in modern medicine, but only poor information and suggestions are available for chronic liver disease. Moreover, the amelioration of management of pediatric chronic hepatopathies, with prompt diagnosis and effective therapy, could lead to a better health care status in adulthood. Treating pediatric patients offers the chance to prevent progressiye liver injury, reducing or delaying the need of liver transplantation. Moreover, prevention programs, aiming for example to reduce alcohol intake or to counteract the epidemic obesity in pediatric setting, should reduce the incidence of alcoholic fatty liver disease (ASH) and NASH in adulthood. At the same time, research programs on pediatric hepatopathies should be strengthened, in order to permit significant advances, in terms of early diagnosis and effective treatment.

\section{Five year view}

In the last years, several advances have been made in the understanding of hepatobiliary physiology with development of more precise approaches to the diagnosis and management of liver disease in infants and children.

These novel discoveries have deeply changed previous knowledge of chronic liver diseases, mainly in terms of pathophysiology and targeted therapies. As known, fibrosis represents the final response of the liver to chronic non-resolving injury. As previously stated, in the liver, fibrosis is mainly due to chronic hepatitis B and C, autoimmune and biliary disorders, metabolic diseases, and, increasingly, NASH. Differently from what is previously described, recent research has demonstrated that fibrosis is not a static phenomenon, but it is the result of a continuous remodeling process, in which various components of the extracellular matrix (ECM), growth factors, proteases and cytokines interact in a complex mechanism. Moreover, a possible regression of fibrosis has been recently described in less advanced rodent and human liver fibrosis models, due to the extraordinary capacity of liver cells to regenerate. But, chronic damage frequently leads to scar tissue and cirrhosis, greatly reducing the potential reversibility of fibrosis. In this view, the early identification of initial fibrosis and possible treatments represent the urgent goal of research in hepatology. Therefore, one of main fields of research in the last few years has been the elucidation of mechanisms of fibrosis in order to identify molecular targets permitting to revert liver fibrosis. 
Assessment of fibrosis is another field of interest, because transaminases and liver function parameters do not correlate with fibrosis and fibrogenesis. Liver biopsy remains the gold standard to detect and assess the staging of fibrosis, but its invasivity, costs and the possibility of sample error, make it, mainly in the pediatric setting, an "imperfect test". More than 2000 studies aiming to assess serological markers or imaging techniques to detect fibrosis have been published in the last five years. The combination of serological markers and elastography techniques seems to increase the diagnostic accuracy, but none of the currently available tests replaced liver biopsy.

In the pathogenetic scenario, the role of gut-liver axis has emerged, opening interesting research fields in understanding the onset and progression of liver diseases, mainly in sclerosing cholangitis and NAFLD, and of treatment modalities. In the near future the characterization and manipulation of intestinal microbiota will represent an attractive field for hepatologist researchers.

Early diagnosis and effective treatment are, especially in children, the mainstay of research in chronic hepatopathies, in order to efficaciously treat liver diseases, reducing the need of liver transplantation in adults, ameliorating quality of life and reducing direct and indirect costs due to liver diseases.

Several advances have also been made in the treatment of hepatic disorders, both metabolic and infectious diseases, with new therapeutic options, which have deeply changed the natural history of several disorders. In the future, definitive therapy, such as targeted enzyme replacement or gene therapy, may be available for certain metabolic disorders. 


\section{Key issues:}

- Identifying the presence of serious liver disease in a pediatric patient at the early stage is of cardinal importance, since it can allow, in many cases, the modification of the therapeutic approach and natural history of the disease.

- The diagnosis of liver disease in children is often delayed; it is important to keep in mind the frequent absence of symptoms or the presence of not-specific signs for several liver diseases.

- Understanding the pathogenetic mechanisms provides the basis for discovering potential early predictors of the disease, suitable noninvasive diagnostic tools and design novel specific treatments for pediatric liver disease.

- Pediatric NAFLD is now considered an increasing public health issue; a large-scale screening in high risk populations, especially among the overweight pediatric patients, should be suggested.

- Chronic viral hepatitis (HBV or HCV) are mild diseases but can lead to cirrhosis and HCC in few cases during life.

- With prompt immunosuppressive therapy the prognosis of patients affected by AIH is generally good, with symptom-free long-term survival in the majority of cases. 


\section{References}

1. Arya G, Balistreri WF. Pediatric liver disease in United States: Epidemiology and impact. J Gastoenterol Hepatol 2002;17:521-525.

2. Blachier M, Leleu H, Peck-Radosavljevic M, Valla D, Roudot-Thoraval F. The burden of liver disease in Europe: A review of available epidemiological data. J Hepatol 2013;58:593-608.

3. Lozano R, Naghavi M, Foreman K, Lim S, Shibuya K, Aboyans V, Abraham J, et al. Global and regional mortality from 235 causes of death for 20 age groups in 1990 and 2010: arsystematic analysis for the Global Burden of Disease Study 2010. Lancet 2012;380:2095-2128.

4. Dezsőfi A, Baumann U, Dhawan A, Durmaz O, Fischler B, Hadzic N, Hierro L, Lacaille F, McLin VA, Nobili V, Socha P, Vajro P, Knisely AS. Liver biopsy in children: position paper of the ESPGHAN Hepatology Committee. J Pediatr Gastroenterol Nutr 2015;60:408-20.

* This position paper shows the role of liver biopsy in children, reporting the indications, contraindications and type of procedures in principal pediatric hepatopathies.

5. Schwimmer JB, Deutsch R, Kahen T, Lavine JE, Stanley C, Behling C. Prevalence of fatty liver in children and adolescents. Pediatrics 2006; 118:1388-1393.

6. Peverill W, Powell LW, Skoien R. Evolving concepts in the pathogenesis of NASH: beyond steatosis and inflammation. Int J Mol Sci. 2014;15:8591-638.

7. Miele L, Valenza V, La Torre G, et al. Increased intestinal permeability and tight junction alterations in nonalcoholic fatty liver disease. Hepatology 2009;49:1877-87.

8. Alisi A, Cianfarani S, Manco M, Agostoni C, Nobili V. Non-alcoholic fatty liver disease and metabolic syndrome in adolescents: pathogenetic role of genetic background and intrauterine environment. Ann Med 2012; 44:29-40.

9. Marzuillo P, Grandone A, Perrone L, Miraglia del Giudice E. Understanding the pathophysiological mechanisms in the pediatric non-alcoholic fatty liver disease: The role of genetics. World J Hepatol. 2015 Jun 18; 7(11): 1439-1443.

10. Dongiovanni P, Anstee QM, Valenti L. Genetic Predisposition in NAFLD and NASH: Impact on Severity of Liver Disease and Response to Treatment. Curr Pharm Des 2013;19:5219-38. 
11. Nobili V, Donati B, Panera N, et al. A Four-Polymorphisms Risk Score Predicts Steatohepatitis in Children With Non-Alcoholic Fatty Liver Disease. J Pediatr Gastroenterol Nutr 2013 Dec 16. [Epub ahead of print].

12. Vajro P, Lenta S, Socha P, et al. Diagnosis of nonalcoholic fatty liver disease in children and adolescents: position paper of the ESPGHAN Hepatology Committee. J Pediatr Gastroenterol Nutr. 2012;54:700-13.

13. Marzuillo P, Grandone A, Perrone L, Miraglia del Giudice E. Controversy in the diagnosis of pediatric non-alcoholic fatty liver disease. World J Gastroenterol. 2015; 21: 6444-6450.

14. Mansoor S, Collyer E, Alkhouri N. A comprehensive review of noninvasive liver fibrosis tests in pediatric nonalcoholic Fatty liver disease. Curr Gastroenterol Rep. 2015;17(6):23.

15. Nobili, V. et al. Accuracy and reproducibility of transient elastography for the diagnosis of fibrosis in pediatric nonalcoholic steatohepatitis. Hepatology 2008; 48: 442-448.

16. Della Corte C, Liccardo D, Ferrari F, Alisi A, Nobili V. Current pharmacotherapy for treating pediatric nonalcoholic fatty liver disease. Expert Opin Pharmacother 2014 ;15:2501-11.

* this review is an interesting overview of etiopathogenisis, diagnosis and treatment of NFLD in children

17. Sanyal A, Chalasani N, Kowdley KV, et al. Pioglitazone, Vitamin E, or Placebo for Nonalcoholic Steatohepatitis. NEngI J Med. 2010; 362: 1675-1685.

18. Chalasani N, Younossi Z, Lavine JE, et al; American Gastroenterological Association; American Association for the Study of Liver Diseases; American College of Gastroenterology. The diagnosis and management of non-alcoholic fatty liver disease: practice guideline by the American Gastroenterological Association, American Association for the Study of Liver Diseases, and American College of Gastroenterology. Gastroenterology 2012;142:1592-609.

19. Lavine JE, Schwimmer JB, Van Natta ML, et al. Effect of vitamin E or metformin for treatment of nonalcoholic fatty liver disease in children and adolescents: the TONIC randomized controlled trial. JAMA 2011;305:1659-68. 
20. Sarkhy AA, Al-Hussaini AA, Nobili V. Does vitamin E improve the outcomes of pediatric nonalcoholic fatty liver disease? A systematic review and meta-analysis. Saudi J Gastroenterol 2014;20:143-53.

21. Nobili V, Bedogni G, Alisi A, Pietrobattista A, Risé P, Galli C, Agostoni C. Docosahexaenoic acid supplementation decreases liver fat content in children with non-alcholic fatty liver disease: double blind randomized controlled clinical trial. Arch Child 2011; 96:350-353.

22. Nobili V, Alisi A, Della Corte C, et al. Docosahexaenoic acid for the treatment of fatty liver: randomised controlled trial in children. Nutr Metab Cardiovasc Dis 2013;23:1066-70.

23. Nobili V, Carpino G, Alisi A, De Vito R, Franchitto A, Alpini G, Onori P, Gaudio E. Role of docosahexaenoic acid treatment in improving liver histology in pediatric nonalcoholic fatty liver disease. PloS One 2014; 9:e88005.

24. Pacifico L, Giansanti S, Gallozzi A, Chiesa C. Long chain omega-3 polyunsaturated fatty acids in pediatric metabolic syndrome. Mini Rev Med Chem. 2014:14(10):791-804.

25. Janczyk W, Socha P, Wierzbicka-Rucińska A, et al. Omega-3 Fatty acids therapy in children with nonalcoholic Fatty liver disease: a randomized controlled trial. J Pediatr. 2015 Jun;166(6):135863.e1-3.

26. Schilling PL, Davis MM, Albanese CT, et al. National trends in adolescent bariatric surgical procedures and implications for surgical centers of excellence. J Am Coll Surg 2008;206:1-12

27. Nobili V, Vajro P, Dezsofi A, Fischler B, Hadzic N, Jahnel J, Lamireau T, McKiernan P, McLin V, Socha P, Tizzard S, Baumann U. Indications and limitations of bariatric intervention in severely obese children and adolescents with and without nonalcoholic steatohepatitis: ESPGHAN Hepatology Committee Position Statement. J Pediatr Gastroenterol Nutr 2015;60:550-61.

It is the first position paper on the use of bariatric surgery in adolescents. Shows the types of interventions used, to date, in the obese adolescents and gives the first indications for the execution of different types of interventions.

28. Mummadi RR, Kasturi KS, Chennareddygair S, et al. Effect of bariatric surgery on nonalcoholic fatty liver disease: systematic review and metaanalysis. Clin Gastroenterol Hepatol 2008;6:1396402. 
29. Hofmann B. Bariatric surgery for obese children and adolescents: a review of the moral challenges. BMC Med Ethics. 2013;14:18.

30. Nobili V, Corte CD, Liccardo D et al. Obalon intragastric balloon in the treatment of paediatric obesity: a pilot study. Pediatr Obes 2014[Epub ahead of print].

31. World Health Organization. Media Centre, Hepatitis B. WHO Website [cited 02.01.2013]. http://www.who.int/mediacentre/factsheets/fs204/en/index.html.

32. Lavanchy D. Hepatitis B. Virus epidemiology, disease burden, treatment, and current and emerging prevention and control measures. J Viral Hepat 2004; 11: 97-107.

33. Luo Z, Li L, Ruan B. Impact of the implementation of a vaccination strategy on hepatitis B virus infections in China over a 20-year period. Int J Infect Dis 2012; 16: e82-88.

34. Liang X, Bi S, Yang W, et al. Epidemiological serosurvey of hepatitis B in China--declining HBV prevalence due to hepatitis B vaccination. Vaccine 2009; 27: 6550-57.

35. Ni YH, Chang MH, Wu JF, Hsu HY, Chen HL, Chen DS. Minimization of hepatitis B infection by a 25-year universal vaccination program. J Hepatol 2012; 57: 730-35.

36. Chen SM, Kung CM, Yang WJ, Wang HL. Efficacy of the nationwide hepatitis B infant vaccination program in Taiwan. J Clin Virol 201 1;52: 11-16.

37. Romano' L, Paladini S, Van Damme P, Zanetti AR. The worldwide impact of vaccination on the control and protection of viral hepatitis B. Dig Liver Dis 2011; 43: S2-S7.

** This review shows the data, to 2011, the efficacy of the vaccine for hepatitis $\mathbf{B}$ worldwide. The implementation of such vaccination programs led globally in a marked decrease in the disease, the rate of carrier and in hepatitis B-related morbidity and mortality.

38. Chang M, Hsu H, Hsu H, Ni Y, Chen J, Chen D. The significance of spontaneous hepatitis B e antigen seroconversion in childhood: with special emphasis on the clearance of hepatitis B e antigen before 3 years of age. Hepatology 1995;22:1387-1392.

39. Chang MH. Prevention of hepatocellular carcinoma by universal vaccination against hepatitis B virus: the effect and problems. Clin Cancer Res 2005;11:7953-7957. 
40. Yu MW, Chang HC, Liaw YF, Lin SM, Lee SD, Liu CJ, et al. Familial risk of hepatocellular carcinoma among chronic hepatitis B carriers and their relatives. J Natl Cancer Inst 2000;92:11591164.

41. Luo Z, Li L, Ruan B. Impact of the implementation of a vaccination strategy on hepatitis B virus infections in China over a 20-year period. Int J Infect Dis 2012;16:e82-e88.

42. Della Corte C, Nobili V, Comparcola D, Cainelli F, Vento S. Management of chronic hepatitis B in children: an unresolved issue. J Gastroenterol Hepatol 2014;29:912-9.

43. Sokal EM, Paganelli M, Wirth S, Socha P, Vajro P, Lacaille F, et al. Management of chronic hepatitis B in childhood: ESPGHAN clinical practice guidelines: consensus of an expert panel on behalf of the European Society of Pediatric Gastroenterology, Hepatology and Nutrition 2013; $59: 814-29$

44. Paganelli M, Stephenne X, Sokal E. Chronic hepatitis B in children and adolescents. J Hepatol 2012; 57: 885-96.

45. Murray KF, Szenborn L, Wysocki J, et al. Randomized, placebo-controlled trial of tenofovir disoproxil fumarate in adolescents with chronic hepatitis B. Hepatology 2012; 56: 2018-26.

46. Chen HL, Lee CN, Chang CH, et al; Taiwan Study Group for the Prevention of Mother-to-Infant Transmission of HBV (PreMIT Study); Taiwan Study Group for the Prevention of Mother-to-Infant Transmission of HBV PreMT Study. Efficacy of maternal tenofovir disoproxil fumarate in interrupting mother-to-infant transmission of hepatitis B virus. Hepatology 2015;62:375-86.

47. Greenup AJ, Tan PK, Nguyen V, et al. Efficacy and safety of tenofovir disoproxil fumarate in pregnancy to prevent perinatal transmission of hepatitis B virus. J Hepatol 2014;61:502-7.

48. Lavanchy D. The global burden of hepatitis C. Liver Int 2009; 29: 74-81.

49. Mack CL, Gonzalez-Peralta RP, Gupta N, Leung D, Narkewicz MR, Roberts EA, Rosenthal P, Schwarz KB; North American Society for Pediatric Gastroenterology, Hepatology, and Nutrition. NASPGHAN practice guidelines: Diagnosis and management of hepatitis $\mathrm{C}$ infection in infants, children, and adolescents. J Pediatr Gastroenterol Nutr. 2012;54:838-55.

50. Bortolotti F, Verucchi G, Camma C, et al. Long-term course of chronic hepatitis C in children: from viral clearance to end-stage liver disease. Gastroenterology 2008;134:1900-7. 
51. European Association for Study of Liver. EASL Clinical Practice Guidelines: management of hepatitis C virus infection. J Hepatol. 2014;60:392-420.

52. Tsubota A, Fujise K, Namiki Y, Tada N. Peginterferon and ribavirin treatment for hepatitis C virus infection. World J Gastroenterol 2011; 17: 419-432.

53. Liang TJ, Ghany MG. Therapy of hepatitis C - back to the future. New Engl J Med 2014;370:20437.

* In this article are the first clinical and laboratory results on use of new drugs for the treatment of hepatitis $\mathrm{C}$ in adults (DAAs: direct-acting antiviral agents).

54. Chang MH, Gordon LA, Fung HB. Boceprevir: a protease inhibitor for the treatment of hepatitis C. Clin Ther 2012; 34: 2021-2038

55. Poordad F, McCone J, Bacon BR, Bruno S, Manns MP, Sulkowski MS, Jacobson IM, Reddy KR, Goodman ZD, Boparai N, Di-Nubile MJ, Sniukiene V, Brass CA, Albrecht JK, Bronowicki JP. Boceprevir for untreated chronic HCV genotype 1 infection. N Engl J Med 2011; 364: 1195-1206

56. Serranti D, Indolfi G, Resti M. New treatments for chronic hepatitis C: an overview for paediatricians. World J Gastroenterol 2014; 20: 15965-15974.

57. Herbst DA, Reddy KR. Sofosbuvir, a nucleotide polymerase inhibitor, for the treatment of chronic hepatitis C virus infection. Expert Opin Investig Drugs 2013; 22: 527-536

58. Gane EJ, Stedman CA, Hyland RH, Ding X, Svarovskaia E, Symonds WT, Hindes RG, Berrey MM. Nucleotide polymerase inhibitor sofosbuvir plus ribavirin for hepatitis C. N Engl J Med 2013; 368: $34-44$.

59. Brennan T, Shrank W. New expensive treatments for hepatitis C infection. JAMA 2014;312:593-4.

60. Ala A, Walker AP, Ashkan K, Dooley JS, Schilsky ML. Wilson'sdisease. Lancet 2007;369:397408.

61. Loudianos G, Gitlin JD. Wilson's disease. Semin Liver Dis 2000;20:353—64.

62. Dhawan A, Taylor RM, Cheeseman P, De Silva P, HatsiyiannakisL, Mieli-Vergani G. Wilson's disease in children: 37-year expe-rience and revised King's score for liver transplantation. Liver Transpl 2005;11:441—8. 
63. Ferenci P, Caca K, Loudianos G, Mieli-Vergani G, Tanner S, Sternlieb I, et al. Diagnosis and phenotypic classification of Wilson disease. Liver Int 2003;23:139-42.

64. Nicastro E, Loudianos G, Zancan L, D’Antiga L, Maggiore G,Marcellini M, et al. Genotypephenotype correlation in Italianchildren with Wilson's disease. J Hepatol 2009;50:555-61.

65. El Balkhi S, Trocello JM, Poupon J, Chappuis P, Massicot F, Girardot-Tinant N, et al. Relative exchangeable copper: a new highly sensitive and highly specific biomarker for Wilson's dis- ease diagnosis. Clin Chim Acta 2011;412:2254-60.

66. Nicastro E, Ranucci G, Vajro P, Vegnente A, Iorio R. Re-evaluation of the diagnostic criteria for Wilson disease inchildren with mild liver disease. Hepatology 2010;52:1948-56.

67. Roberts EA, Schilsky ML. American Association for Study of Liver Diseases (AASLD). Diagnosis and treatment of Wilson disease: an update. Hepatology 2008;47:2089-111.

68. European Association for the Study of the Liver. EASL Clinical Practice Guidelines: Wilson's disease. J Hepatol 2012;56:671—85.

69. The Human Gene Mutation Database. http://www.hgmd.cf. ac.uk/ac/gene.php?gene=ATP7B.

70. Schilsky ML. Long-term outcome for Wilson disease: $85 \%$ good. Clin Gastroenterol Hepatol 2013;16:1542-3565.

71. Mieli-Vergani G, Vergani D. Autoimmune hepatitis. Nat Rev Gastroenterol Hepatol 2011;8:320-9.

72. Krawitt EL. Discrimination of autoimmune hepatitis: autoantibody typing and beyond. J Gastroenterol. 2011;46:39-41.

73. Mieli-Vergani G, Vergani G. Autoimmune hepatitis. J Hepatol 2011;55:171-82

74. Gregorio GV, Portmann B, Reid F, Donaldson PT, Doherty DG, McCartney M, et al. Autoimmune hepatitis in childhood: a 20-year experience. Hepatology 1997;25:541-7.

75. Manns MP, Czaja AJ, Gorham JD, Krawitt EL, Mieli-Vergani G, Vergani D, et al. Diagnosis and management of autoimmune hepatitis. Hepatology 2010;51:2193-213.

76. Radhakrishnan KR, Alkhouri N, Worley S, Arrigain S, Hupertz V, Kay M, et al. Autoimmune hepatitis in children--impact of cirrhosis at presentation on natural history and long-term outcome. Dig Liver Dis 2010;42:724-8.

77. Vergani D, Mieli-Vergani G. Pharmacological management of autoimmune hepatitis. N Engl J Med 
78. Aw MM, Dhawan A, Samyn M, Bargiota A, Mieli-Vergani G. Mycophenolate mofetil as rescue treatment for autoimmune liver disease in children: a 5-years follow up. J Hepatol 2009;51:156-60.

79. Schramm C, Lohse AW. Role of mycophenolate mofetil in the treatment of autoimmune hepatitis. J Hepatol 2011;55:510-1.

80. Tannous MM, Cheng J, Muniyappa K, Farooq I, Bharara A, Kappus M, et al. Use of tacrolimus in the treatment of autoimmune hepatitis: a single centre experience. Aliment Pharmacol Ther 2011;34:405-7.

81. Damoiseaux JC, van Breda Vriesman PJ. Cyclosporin A-induced autoimmunity: the result of defective de novo T-cell development. Folia Biol 1998;44:1-9.

82. Wiegand J, Schuler A, Kanzler S, Lohse A, Beuers U, Kreisel W, et al. Budesonide in previously untreated autoimmune hepatitis. Liver Int 2005;25:927-34.

83. Csepregi A, Rocken C, Treiber G, Malfertheiner P. Budesonide induces complete remission in autoimmune hepatitis. World J Gastroenterol 2006;12:1362-6.

84. Woynarowski M, Nemeth A, Baruch Y, et al; European Autoimmune Hepatitis-Budesonide Study Group. Budesonide versus prednisone with azathioprine for the treatment of autoimmune hepatitis in children and adolescents, J Pediatr 2013;163:1347-53.

85. Manns MP, Woynarowski M, Kreisel W, et al; European AIH-BUC-Study Group. Budesonide induces remission more effectively than prednisone in a controlled trial of patients with autoimmune hepatitis. Gastroenterology. 2010;139:1198-206.

86. Floreani A, Liberal R, Vergani D, Mieli-Vergani G. Autoimmune hepatitis: Contrasts and comparisons in children and adults - a comprehensive review. J Autoimmun. 2013 Oct;46:7-16.

87. Gassert DJ, Tanaka HGK, Reinus JF. Corticosteroid-Responsive Cryptogenic Chronic Hepatitis: Evidence for Seronegative Autoimmune Hepatitis. Dig Dis Sci 2007;52:2433-7. 
Financial and competing interests disclosure

The authors have no relevant affiliations or financial involvement with any organization or entity with a financial interest in or financial conflict with the subject matter or materials discussed in the manuscript. This includes employment, consultancies, honoraria, stock ownership or options, expert testimony, grants or patents received or pending, or royalties.

Table 1. Most frequent cause of liver disease in children according to age group

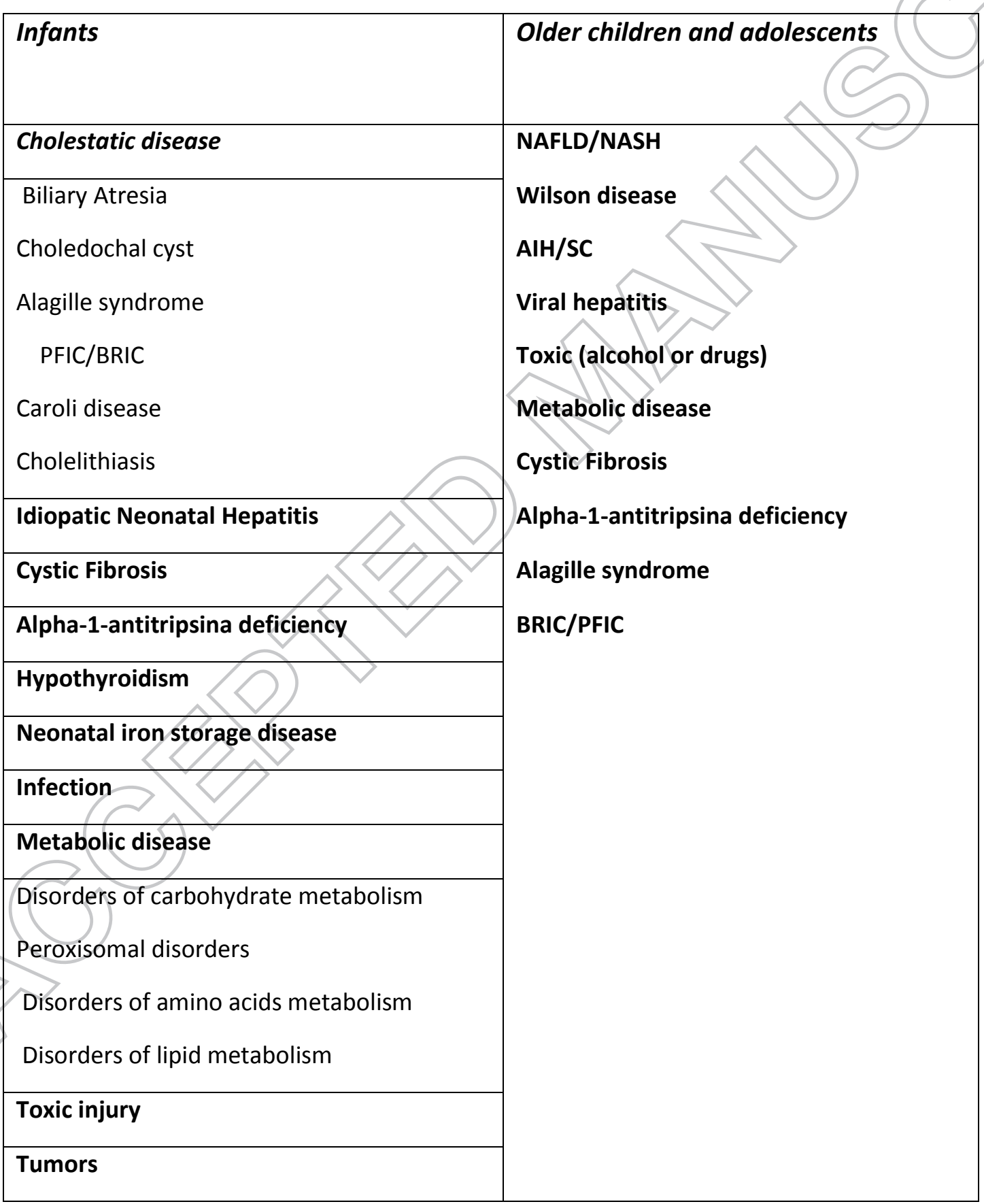


Table 2. Clinical and laboratory red flags to suspect a liver disease

\begin{tabular}{|l|l|}
\hline Signs & Symptoms \\
\hline Hepatomegaly & $\begin{array}{l}\text { Non-specific symptoms, such as } \\
\text { asthenia, abdominal pain, anorexia } \\
\text { Hepatosplenomegaly }\end{array}$ \\
Jaundice & Pruritus \\
Gastrointestinal bleeding & \\
Facies with dysmorphic features \\
Spider nevi \\
Xanthomas \\
Dark and foamy urine \\
Acholic stool
\end{tabular}

